OPEN ACCESS

Edited by:

Zhenxiang Cheng,

University of Wollongong, Australia

Reviewed by:

Guangzhao Wang,

Yangtze Normal University, China

Shakeel Ahmad Khandy

National Taiwan University, Taiwan

*Correspondence:

Chunling Tian

tclswn@163.com

Specialty section:

This article was submitted to Computational Materials Science,

a section of the journal

Frontiers in Materials

Received: 08 February 2020 Accepted: 17 April 2020

Published: 16 June 2020

Citation:

Ke C, Tian C and Gan Y (2020) Tailoring the Band Gap in Codoped GaN Nanosheet From First Principle

Calculations. Front. Mater. 7:124

doi: 10.3389/fmats.2020.00124

\section{Tailoring the Band Gap in Codoped GaN Nanosheet From First Principle Calculations}

\author{
Changjiao $\mathrm{Ke}^{1}$, Chunling Tian ${ }^{1 *}$ and Yundan Gan ${ }^{2}$ \\ ${ }^{1}$ School of Physical Science and Technology, Southwest University, Chongqing, China, ${ }^{2}$ Xi'an Modern Chemistry Research \\ Institute, Xi'an, China
}

To develop an efficient photocatalyst with intense visible light absorption and high charge mobility is important but still remains a problem. In this work, we have explored the electronic properties of C-monodoped, C-Ge, and C-Sn codoped GaN nanosheets by the hybrid density functional theory in order to find the excellent photocatalytic materials. Results indicate the monodoping of $\mathrm{C}$ introduces unoccupied impurity states inside the band gap that serve on recombination centers. Thus, the $\mathrm{C}$ monodoping is not suitable to ameliorate visible light absorption. Moreover, the C-Ge and C-Sn codoping not only successfully reduce the band gap of nanosheet GaN but also avoid the unoccupied impurity states. The charge-compensated C-Ge and C-Sn codoped GaN nanosheets are energetically favorable for hydrogen evolution but not insufficient to produce oxygen, indicating that they could serve as Z-scheme photocatalysts. In particular, the minimum defect formation energy of C-Ge is negative and lowest. The C-Ge codoped GaN system has dynamic stability. So, the C-Ge codoped nanosheet GaN is one of the most prospective candidates for the decomposition of hydrogen from water.

Keywords: photocatalysis, hybrid density functional, doped, band structural calculation, GaN nanosheet

\section{INTRODUCTION}

Gallium nitride $(\mathrm{GaN})$ has attracted considerable interest in recent years for its excellent photoelectric properties. With a large band gap (3.04 eV) (Bastos et al., 2018), GaN is widely used as short-wavelength light-emitting diodes (Ha et al., 2007) and room-temperature laser diodes (Feng et al., 2017). The conduction band edge of bulk $\mathrm{GaN}$ is at $0.5 \mathrm{eV}$ higher than the redox potential of $\mathrm{H}^{+} / \mathrm{H}_{2}$, which implies that the GaN can decompose hydrogen from water (Maeda et al., 2005; Kida et al., 2006). Its wide band gap, however, makes GaN photochemically active only under UV light irradiation. In order to extend the visible light response of $\mathrm{GaN}$, some efforts have been made based on band gap engineering strategies. It was found that $\mathrm{Cr}-\mathrm{O}$ codoping significantly narrows the band gap of GaN, but this leads to an intermediate band entering the band gap (Pan et al., 2010), which increases the probability of carrier capture. Similar results were obtained for V-O codoped GaN (Meng et al., 2012).

According to the recent theoretical calculations (Freeman et al., 2006), the monolayer GaN has a honeycomb structure similar to that of graphene (Castro Neto et al., 2009), and boron nitride nanosheets (Golberg et al., 2010). These two-dimensional (2D) atomic-layer nanomaterials are interesting topics for their picturesque properties, which would have advantages over bulk structures in many cases (Onen et al., 2016). Particularly, the 2D GaN has a large specific surface area and shorter carrier migration distance etc., and it can remain in high-temperature 
environments (Sun et al., 2017). The unique properties of nanosheets $\mathrm{GaN}$ motivate our research into whether a tunable band gap could be obtained via chemical modification. In this work, the C-monodoping and $\mathrm{C}-\mathrm{Ge}$ and $\mathrm{C}$-Sn codoping were used to modulate the band structure of $\mathrm{GaN}$ nanosheet. We focused our studies on the structure, electronic properties, and minimum defect formation energies of the doped GaN nanosheets.

\section{COMPUTATIONAL METHOD}

Our first-principles calculations were executed by using density functional theory (DFT) within generalized gradient approximation (GGA) (White and Bird, 1994) parameterized by Perdew, Burke, and Ernzerhof (PBE) (Ernzerhof and Scuseria, 1999), as realized in VASP code (Kresse and Furthmüller, 1996; Kresse and Joubert, 1999). Projector augmented wave (PAW) (Blöchl, 1994) potentials were employed to treat interactions between ions and valance electrons. The electron configuration of $\mathrm{N}\left(2 \mathrm{~s}^{2} \mathrm{p}^{3}\right), \mathrm{Ga}\left(3 \mathrm{~d}^{10} 4 \mathrm{~s}^{2} 4 \mathrm{p}^{1}\right), \mathrm{C}\left(2 \mathrm{~s}^{2} 2 \mathrm{p}^{2}\right), \mathrm{Ge}\left(3 \mathrm{~d}^{10} 4 \mathrm{~s}^{2} 4 \mathrm{p}^{2}\right)$, and Sn $\left(5 s^{2} 5 p^{2}\right)$ were chosen to construct the potential function, respectively. All the calculations were carried out with a planwave cutoff energy of $550 \mathrm{eV}$. We adopted vacuum space of $20 \AA$ along the $\mathrm{z}$-direction in order to wipe out the interaction between the periodic images. All the atomic structure energy convergence tolerance for self-consistent iteration was set to $1.0 \times 10^{-5} \mathrm{eV}$, and the convergence criteria force was set to $0.01 \mathrm{eV}^{-1}$. The Brillouin zone integrations were approximated with a $9 \times 9 \times$ $1 k$-point (Monkhorst and Pack, 1976) for the GaN nanosheet structure relaxation and electronic properties calculations. The GGA-PBE algorithm will underrate the band gap value of the semiconductor. The accurate electronic structure was calculated by the Heyd-Scuseria-Ernzerhof (HSE06) (Heyd et al., 2003; Heyd and Scuseria, 2004) hybrid functional method. The HSE06 expression for the exchange-correlation energy was written a:

$$
E_{X C}^{H S E}=\chi E_{X}^{S R}(\mu)+(1-\chi) E_{X}^{P B E, S R}(\mu)+E_{X}^{P B E, L R}(\mu)+E_{C}^{P B E}
$$

The exchange-correlation energy includes the short- and longranged parts. Here, the SR is the short-range parts, and LR stands for the long-ranged. $\chi$ represents the mixing coefficient and is set to 0.25 . $\mu$ indicates the screening parameter and is set to 0.2 . In addition, the total density of states (TDOS) and the projected density of states (PDOS) are calculated by the tetrahedral method with Blöchl correction (Blöchl et al., 1994).

\section{RESULTS AND DISCUSSION}

\subsection{Pure GaN Nanosheet}

The GaN nanosheet is the (001) facet of the optimized bulk, which has a honeycomb graphite structure. This structure is shown in Figures 1A,B. The Ga-N bond length is $1.849 \AA$ and is smaller than in the bulk GaN case (1.966 $\AA$ ) (Sahin et al., 2009). Since the $\mathrm{sp}^{2}$ bond is stronger than the $\mathrm{sp}^{3}$ bond, the bond length in the $\mathrm{GaN}$ nanosheet becomes shorter. The Figure 1C displays the band structure of the GaN nanosheet with the band gap of $3.41 \mathrm{eV}$ using the HSE06 functional. The VBM is located at the $\mathrm{k}$ point while the $\mathrm{CBM}$ is at the $\Gamma$ point, which means that the GaN nanosheet is the indirect band gap semiconductor. According to the Figure 1D, the GaN nanosheet has nonmagnetic semiconducting character, and the VBM and CBM are predominantly derived from N 2p, Ga 4p, and Ga 3d orbits. The hybridization between the N 2p, Ga 4p, and Ga 3d orbits are located at the bottom of the conduction band. The bond length $d_{G a-N}$, lattice constant $a$, and band gaps calculated by the PBE and HSE06 functionals are given in Table 1. Our theoretical data have exhibited good agreement with previous studies (Maruska and Tietjen, 1969; Armstrong et al., 2005; Zeyan et al., 2008; Sahin et al., 2009; Xia et al., 2013; Bastos et al., 2018), which indicates that the calculation methods in this study are reasonable. It can be found that the PBE algorithm will underestimate the band gap of GaN nanosheet. Therefore, the HSE06 algorithm is adopted to study the electronic structures of the GGA-PBE optimized phases.

\subsection{Monodoping in the GaN Nanosheet}

In this section, we studied the effect of $\mathrm{C}$ doping on the electronic properties of the nanosheet $\mathrm{GaN}$. For $\mathrm{GaN}_{1-x} \mathrm{C}_{x}$, the nanosheets (with $\mathrm{x}=0.125$ ) were modeled by replacing aneutral $\mathrm{N}$ atom (A site in Figure 1A) with a $\mathrm{C}$ atom in the $2 \times 2 \times 1$ supercell of the $\mathrm{GaN}$ nanosheet. Because the charge number of the doping system is less than that of the pure system, we considered the spin-polarization effect in the calculation.

For the $\mathrm{C}$ modoped $\mathrm{GaN}$ nanosheet, the bond distance between the $\mathrm{C}$ atom and the nearest-neighboring $\mathrm{Ga}$ atom is $1.911 \AA$, which is close to the bond distance (1.849 $\AA$ ) of Ga-N in nanosheet $\mathrm{GaN}$. The minor difference may be explained by the ionic radius difference between $\mathrm{C}$ and $\mathrm{N}$ atom. The DOS and band structure of $\mathrm{GaN}$ nanosheets with the $\mathrm{C}$ doping presented in Figures 2A,B. For both $\mathrm{C}$ monodoped systems, the VBM is mainly contributed by mixing of the $\mathrm{N} \mathrm{2p}, \mathrm{C} 2 \mathrm{p}, \mathrm{Ga} 4 \mathrm{p}$, and Ga 3d states, whereas the CBM is primarily contributed by the mixing of the between Ga $4 \mathrm{p}, \mathrm{N} 2 \mathrm{p}, \mathrm{Ga} 3 \mathrm{~d}$, and C $2 \mathrm{p}$ states. C dopant introduces the unoccupied impurity states on the spin down direction. The impurity state formed by a strong hybrid of C $2 p$ and $\mathrm{Ga} 4 \mathrm{p}$ is very close to the conduction band of GaN. The generation of the impurity state may be due to the fact that the $\mathrm{C}$ and $\mathrm{N}$ are elements of the same cycle, and the $\mathrm{p}$ orbital energy of $\mathrm{C}$ is thus higher than that of $\mathrm{N}$. Electrons in the valence band can easily migrate to the conduction band through these impurity states. Although the band gaps for $\mathrm{C}$ doping $\mathrm{GaN}$ nanosheet decrease to $1.84 \mathrm{eV}$, the monodoped system has partially unoccupied impurity states at top of Fermi level. These impurity states can facilitate electron-hole recombination and have destructive influence on the photocatalytic activity. It indicates that $\mathrm{C}$ monodoped $\mathrm{GaN}$ nanosheet is not suitable for a visible light photocatalyst.

\subsection{Codoping in the GaN Nanosheet}

To overcome the unoccupied impurity states, the anion-cation passivated codoping was utilized to maintain the charge balance (Gai et al., 2009). In this section, we have discussed the influence of codoping $\mathrm{C}-\mathrm{Ge}$ and $\mathrm{C}-\mathrm{Sn}$ on the electronic properties of the supercell $2 \times 2 \times 1 \mathrm{GaN}$ nanosheet. The $\mathrm{C}$ atom substitutes 
A
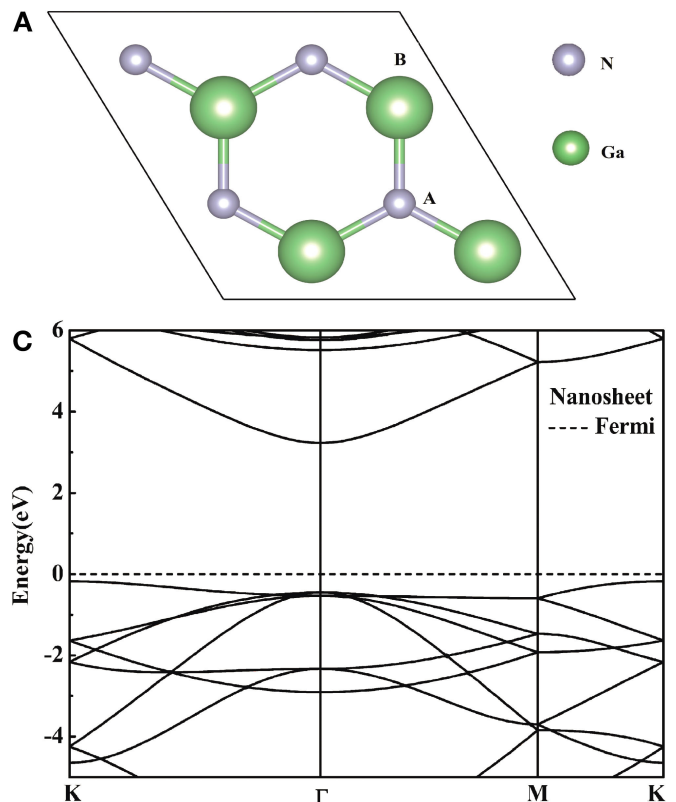

B

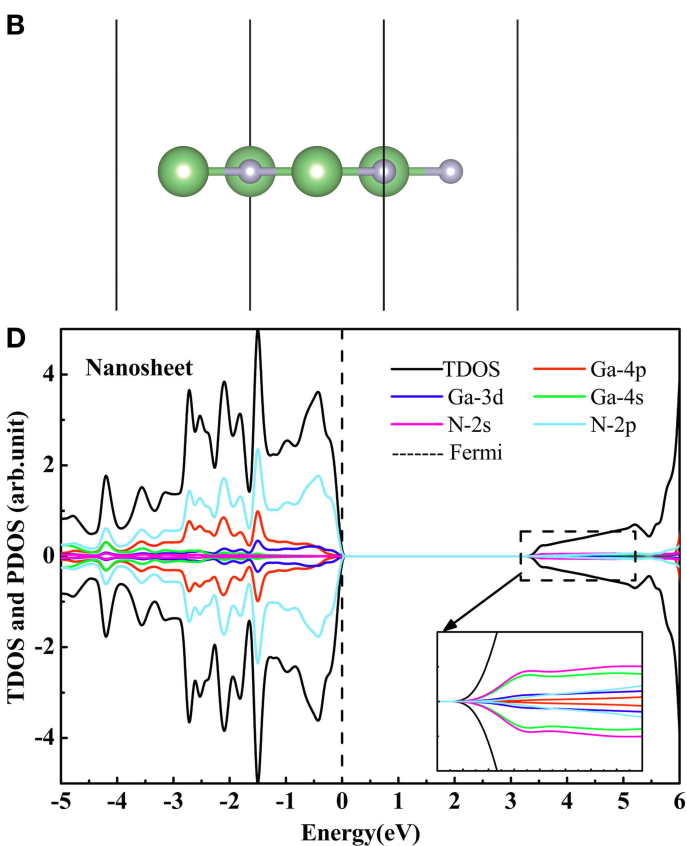

FIGURE 1 | The top (A) and side (B) views of GaN nanosheet. The HSEO6 calculated band structure (C) and DOS (D) of the pure GaN nanosheet.
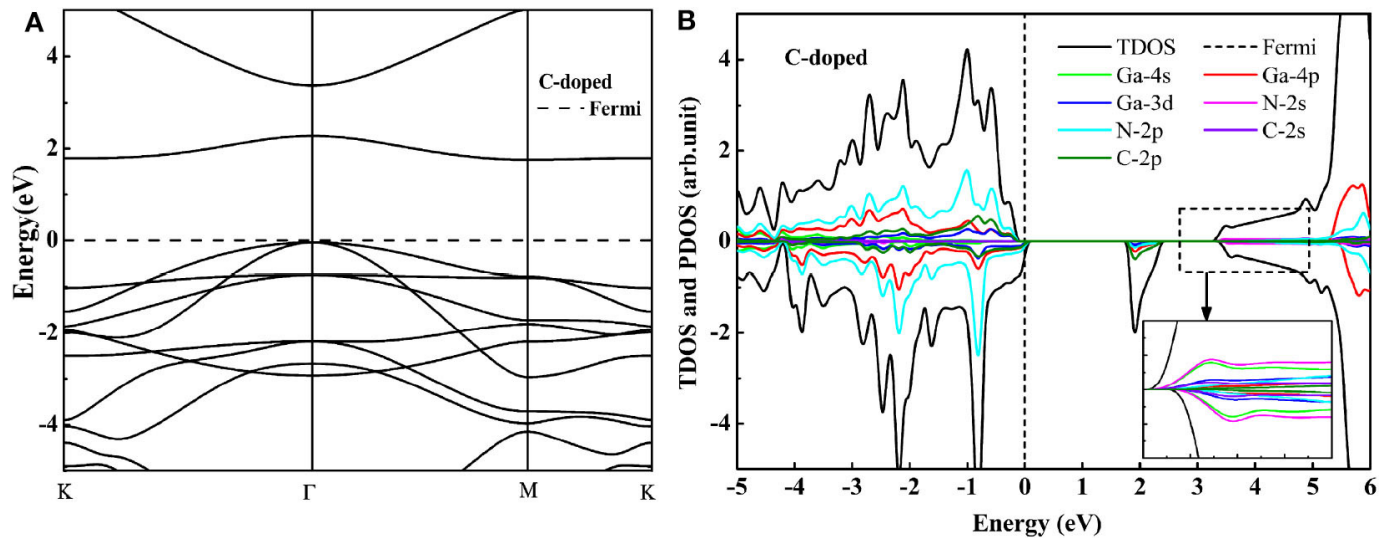

FIGURE 2 | The HSE06 calculated band structure (A) and DOS (B) of the C monodoped GaN nanosheet.

TABLE 1 | The obtained lattice constant $a$, bond length $d_{G a-N}$, and band gap $E_{g}$ with the PBE and HSEO6 functional.

\begin{tabular}{|c|c|c|c|c|c|}
\hline Structure & & $a(\AA ̊)$ & $d_{G a-N}$ & HSE06- $E_{g}(\mathrm{eV})$ & PBE- $E_{g}(\mathrm{eV})$ \\
\hline Nanosheet & Other work & 3.216 (Xia et al., 2013) & 1.851 (Xia et al., 2013) & 3.44 (Armstrong et al., 2005) & 2.27 (Sahin et al., 2009) \\
\hline
\end{tabular}

the $\mathrm{N}$ atom (A site in Figure 1A), and the Ge (or $\mathrm{Sn}$ ) atom substitutes the $\mathrm{Ga}$ atom (B site in Figure 1A), respectively. For $\mathrm{C}-\mathrm{Ge}$ and $\mathrm{C}-\mathrm{Sn}$ codoping, the N-Ge bond length is $1.829 \AA$, and the N-Sn bond distance is $2.00 \AA$, decreasing $0.02 \AA$, and increasing $0.151 \AA$, respectively, with comparison with the N-Ga bond length (1.849 $\AA$ ). The C-Ge bond length is $1.810 \AA$ and the C-Sn bong length is $1.986 \AA$. The DOS and band structure for the $\mathrm{C}-\mathrm{Ge}$ are plotted in Figure 3. According to the DOS, we found that the VBM is dominated by C 2p, Ga 4p, N 2p, and $\mathrm{Ga} 3 \mathrm{~d}$ states, whereas the CBM is dominated by $\mathrm{N} 2 \mathrm{~s}, \mathrm{Ge}$ $4 \mathrm{~s}, \mathrm{Ga} 4 \mathrm{~s}$, and $\mathrm{N} 2 \mathrm{p}$ states. Since the band structures display that the VBM and CBM are located at the $\Gamma$ point, the $\mathrm{C}-\mathrm{Ge}$ system is the direct band gap semiconductor. The calculated band gap of $\mathrm{C}-\mathrm{Ge}$ codoped $\mathrm{GaN}$ nanosheet is $1.83 \mathrm{eV}$. The 

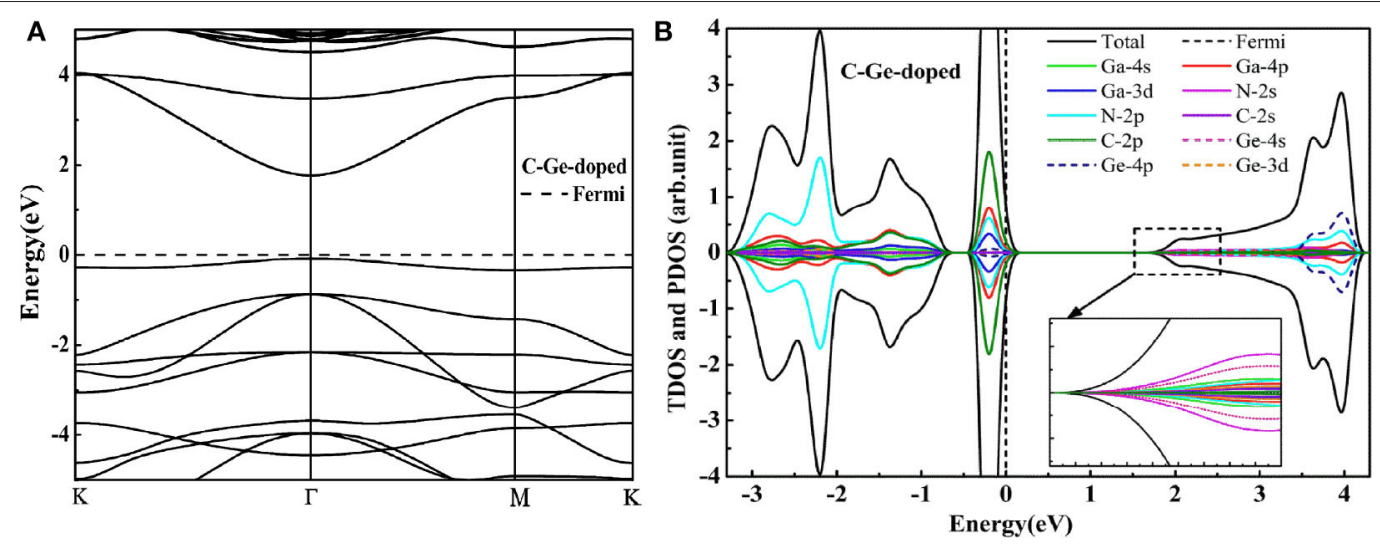

FIGURE 3 | The HSE06 calculated band structure (A) and DOS (B) of the C-Ge codoped GaN nanosheet.
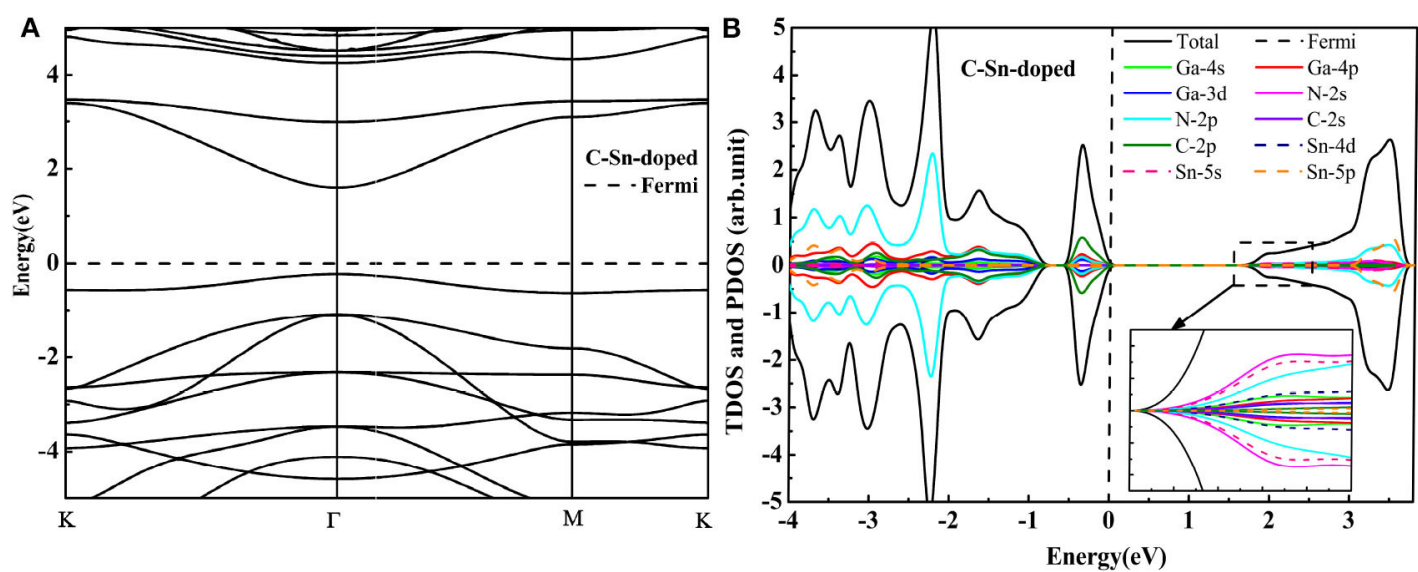

FIGURE 4 | The HSE06 calculated band structure (A) and DOS (B) of the C-Sn codoped GaN nanosheet.

DOS of C-Sn doping is plotted in Figure 4B. It indicates that the VBM is dominated by $\mathrm{C} 2 \mathrm{p}, \mathrm{Ga} 4 \mathrm{p}, \mathrm{N} 2 \mathrm{p}$, and $\mathrm{Ga} 3 \mathrm{~d}$, whereas the CBM is dominated by $\mathrm{Sn} 5 \mathrm{~s}, \mathrm{~N} 2 \mathrm{p}, \mathrm{Ga} 4 \mathrm{p}$, and $\mathrm{Sn} 4 \mathrm{~d}$. These configurations have strong hybridization at the top of the valence band and at the bottom the conduction band. According to Figure 4A, the calculated band gap of C-Sn codoped $\mathrm{GaN}$ nanosheet is $1.85 \mathrm{eV}$. The interesting phenomenon is that the impurity states are completely occupied for codoped GaN nanosheets.

As discussed above, those results indicate that the nanosheet GaN changes from an indirect band gap to a direct band gap by $\mathrm{C}-\mathrm{Ge}$ and $\mathrm{C}-\mathrm{Sn}$ codoping. The nanosheet $\mathrm{GaN}$ band gap decreases from 3.41 to $1.83 \mathrm{eV}$ in the $\mathrm{C}-\mathrm{Ge}$ codoping case and to $1.85 \mathrm{eV}$ in the C-Sn codoping one. Smaller band gap values and a direct band gap are beneficial for excited state electronics, as they allow for greater ease with which to jump to the conduction band. At the same time, the smaller band gap value is favorable for moving the absorption spectrum to the visible light region. Therefore, the $\mathrm{C}-\mathrm{Ge}$ and $\mathrm{C}-\mathrm{Sn}$ codoped nanosheets $\mathrm{GaN}$ are likely to remarkable candidates for visible light photocatalyst.

\subsection{Defect Formation Energy}

We have calculated the defect formation energy $\left(E_{f}\right)$ for evaluating the stability of doping system in each case. The defect formation energy is defined as (Van de Walle and Neugebauer, 2004; Castleton et al., 2006):

$$
E_{f}=E(\text { doped })-E(G a N)-\sum_{i} n_{i} \mu_{i}
$$

Here, $E($ doped $)$ and $E(G a N)$ indicate the total energies of doped and pure nanosheet $\mathrm{GaN}$, respectively. $n_{i}$ represents the number of the atoms that has been added to $\left(n_{i}>0\right)$ or removed from $\left(n_{i}<0\right)$ the pure system for the construction doped nanosheet $\mathrm{GaN}$, while $\mu_{i}$ is the chemical potential of the element $i$, and its value $\mu_{i}$ rests with the synthetic environment

$$
\mu_{G a}+\mu_{N}=\mu_{G a N(\text { nanosheet })}
$$

Here, $\mu_{G a N \text { (nanosheet) }}$ represents the chemical potential, which is equal to the energy of the unit cell. As the chemical potential of 


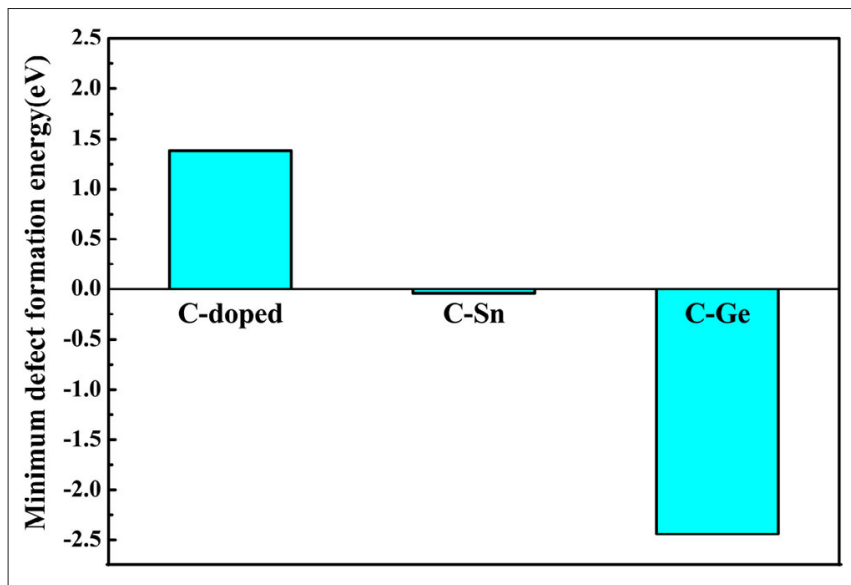

FIGURE 5 | The obtained minimum defect formation energies for monodoped and codoped GaN nanosheets.

Ga and $\mathrm{N}$ cannot exceed that of the Ga bulk $\mu_{G a(b u l k)}$ and $\mathrm{N}$ gas $\mu_{N(\text { gas })}$, the following rules must be obeyed

$$
\begin{gathered}
\mu_{G a}^{\min } \leq \mu_{G a} \leq \mu_{G a(\text { bulk })} \\
\mu_{N}^{\text {min }} \leq \mu_{N} \leq \mu_{N(\text { gas })}
\end{gathered}
$$

To form the nanosheet GaN naturally, the minima of $\mu_{G a}$ and $\mu_{N}$ will satisfy

$$
\begin{aligned}
& \mu_{G a}^{m i n}=E\left(G a_{n} N_{n}\right)-E\left(G a_{n-1} N_{n}\right) \\
& \mu_{N}^{m i n}=E\left(G a_{n} N_{n}\right)-E\left(G a_{n} N_{n-1}\right)
\end{aligned}
$$

Here, $E\left(G a_{n} N_{n}\right)$ is the total energy of perfect system with $\mathrm{n}$ primitive cells, $E\left(G a_{n-1} N_{n}\right)$ the total energy with Ga defect, and $E\left(G a_{n} N_{n-1}\right)$ the total energy with $\mathrm{N}$ defect. We set the $\Delta \mu_{G a}=\mu_{G a}-\mu_{G a(b u l k)}$ and $\Delta \mu_{N}=\mu_{N}-\mu_{N(g a s)}$. In this article, only the minimum defect formation energies of doped $\mathrm{GaN}$ nanosheets have been estimated; e.g., the defect formation energy take the minimum value when $\mu_{G a}$ equals $\mu_{G a(b u l k)}$. The minimum formation energies for doping GaN nanosheets are shown in Figure 5, and the results are outlined in Table 2.

From the above discussion, we know that the minimum defect formation energy for the $\mathrm{C}$ monodoping is positive, which means that the $\mathrm{C}$ monodoping is more difficult to form because the electronegativity of $\mathrm{C}$ is less than that of $\mathrm{N}$. The C-Ge and C-Sn codoping minimum defect formation energies are significantly lower than $\mathrm{C}$ monodoping. Importantly, the minimum defect formation energy of the C-Ge codoped is negative and lowest, which is greatly reduced by the opposite charge states of CGe atoms.

\subsection{Band Edge Alignment}

It is well-known that an excellent photocatalyst should not only have an appropriate band gap but also suitable band edge positions, i.e., The water reduction level and oxidation level must
TABLE 2 | The calculated band gaps $\left(E_{g a p}\right)$, minimum defect formation energies $\left(E_{f}\right)$, and chemical potentials of $\mathrm{N}$ and $\mathrm{Ga}$.

\begin{tabular}{lcccc}
\hline Structure & $\mathbf{E}_{\text {gap }}(\mathbf{e V})$ & $\mathbf{E}_{\boldsymbol{f}}(\mathbf{e V})$ & $\Delta \boldsymbol{\mu}_{\mathrm{Ga}}(\mathbf{e V})$ & $\Delta \boldsymbol{\mu}_{\mathbf{N}}(\mathrm{eV})$ \\
\hline $\mathrm{C}(\mathrm{x}=0.125)$ & 1.84 & 1.39 & -0.36 & -0.36 \\
$\mathrm{C}-\mathrm{Sn}$ & 1.85 & 0.55 & -0.36 & -0.36 \\
$\mathrm{C}-\mathrm{Ge}$ & 1.83 & -1.71 & -0.36 & -0.36
\end{tabular}

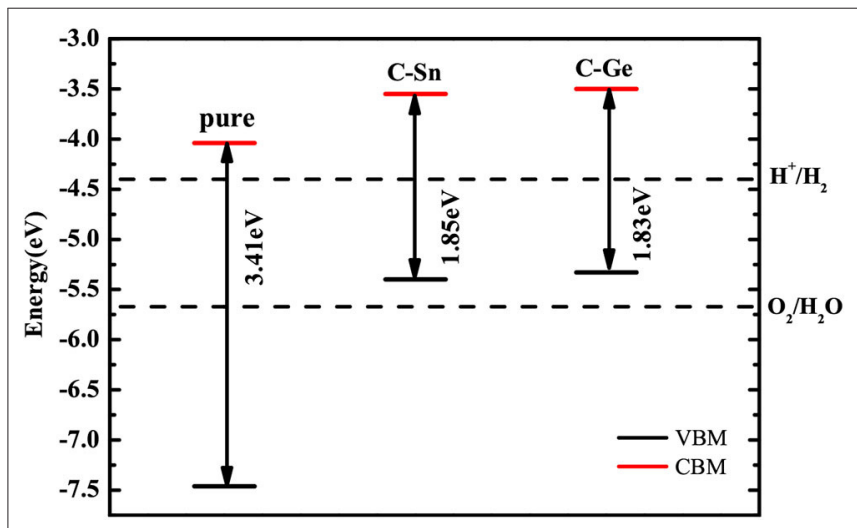

FIGURE 6 | The calculated VBM and CBM positions of pure and codoped GaN nanosheets with respect to the water redox potentials.

lie between the CBM and VBM in order to thermodynamically allow for both the hydrogen evolution reaction and oxygen evolution. On the basis of the band edge positions, some photocatalysts are suitable for only hydrogen evolution, and some photocatalysts are good for only oxygen evolution, which is called the Z-scheme photocatalysis system. Here, we have considered the situation of the standard water reduction level $(-4.44 \mathrm{eV})$ and oxidation level $(-5.67 \mathrm{eV})$ with respect to the vacuum level (Chakrapani et al., 2007). A large vacuum space has been used to calculate the absolute position of the band edge for quantitatively comparing with the water redox potentials. As the calculated vacuum level is a relative value, we have shifted the energy value of the CBM (VBM) for each system by subtracting this value to obtain the absolute band edge position.

The calculated positions of the CBM and VBM of the pure and doped GaN nanosheets with respect to the standard hydrogen electrode potentials are indicated in Figure 6. Compared to the band edge potentials of pure $\mathrm{GaN}$, both $\mathrm{CB}$ and $\mathrm{VB}$ edges of codoped systems are increased. The calculated CBM and VBM of $\mathrm{C}-\mathrm{Ge}(\mathrm{C}-\mathrm{Sn})$ codoped nanosheet are located at $-3.50 \mathrm{eV}(-3.55$ $\mathrm{eV})$ and $-5.33 \mathrm{eV}(-5.40 \mathrm{eV})$, respectively. The CBM of C$\mathrm{Ge}(\mathrm{C}-\mathrm{Sn})$ is $0.90 \mathrm{eV}(0.85 \mathrm{eV})$ above the water reduction level, and the VBM of C-Ge $(\mathrm{C}-\mathrm{Sn})$ is $0.34 \mathrm{eV}(0.27 \mathrm{eV})$ above the water oxidation level. Thus, the C-Sn and C-Ge codoped GaN nanosheets are energetically favorable for hydrogen evolution but not insufficient to produce oxygen, indicating that they could serve as Z-scheme photocatalysts.

\subsection{Optical Properties}

Optical properties play a critical role in evaluating the performance of photocatalysts in the visible light region. Thus, 


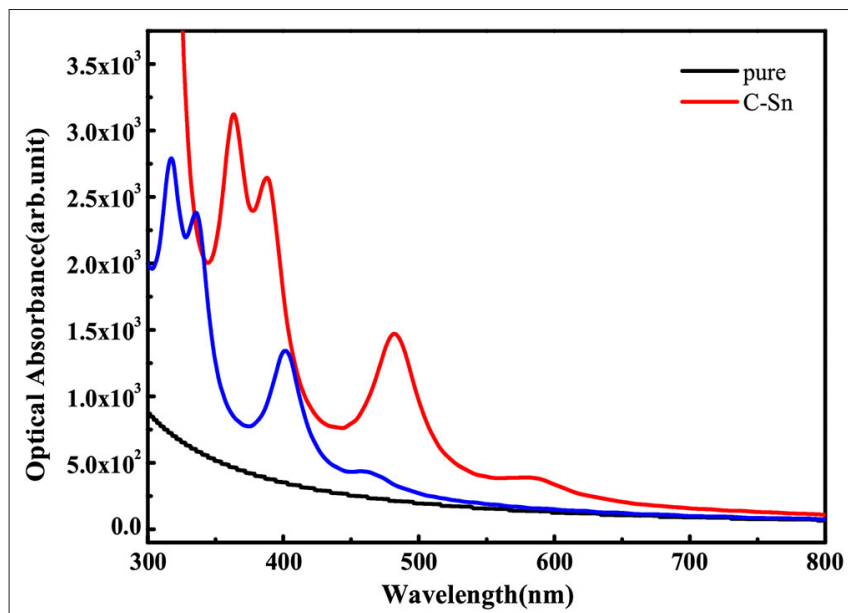

FIGURE 7 | The optical absorption spectra of pure and codoped GaN nanosheets.

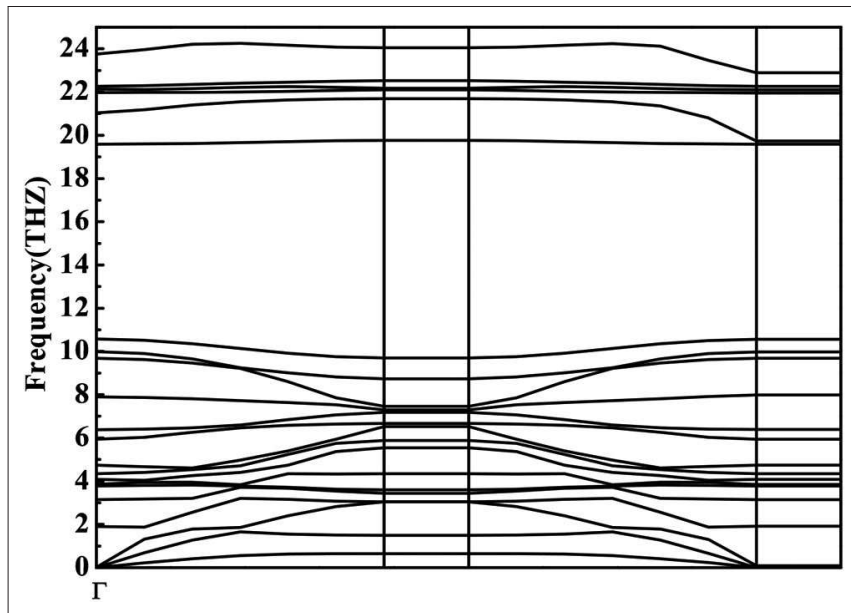

FIGURE 8 | Phonon dispersion for C-Ge codoped GaN nanosheet.

we have investigated the optical absorption spectra of the pure and codoped GaN nanosheets. The absorption coefficient $\alpha(\omega)$ satisfies the following relation (Onen et al., 2016)

$$
\alpha(\omega)=\sqrt{2} \omega \sqrt{\sqrt{\varepsilon_{1}^{2}(\omega)+\varepsilon_{2}^{2}(\omega)}-\varepsilon_{1}(\omega)}
$$

where $\varepsilon_{1}(\omega)$ is the real part that is obtained using the KramersKronig transformation, and $\varepsilon_{2}(\omega)$ is the imaginary part, which has been calculated by summing over a large amount of empty states. Figure 7 shows the obtained absorption coefficient of pure and codoped GaN nanosheets. It can be seen that the C-Sn and $\mathrm{C}-\mathrm{Ge}$ codoped can harvest a longer wavelength of visible light spectrum compared to the pure GaN nanosheet for visible light photocatalysis, which can be attributed to the reasonable band gap reduction.

\subsection{Dynamical Stability}

Phonon calculations provide a criterion for the stability of one structure and indicate, through soft modes, structural instability (Ahmad Khandy et al., 2019; Xuan et al., 2019; Ahmad Khandy and Chai, 2020). We therefore calculated phonon dispersion curves for the codoped GaN nanosheet. The $\mathrm{C}-\mathrm{Ge}$ codoping system was considered, as it has a negative formation energy and can be stably formed. Figure 8 shows the phonon dispersion curves (PDCs) of C-Ge codoping system obtained using GGA scheme, which contains 24 phonon branches composed of $\mathrm{Ga}_{3} \mathrm{GeN}_{3} \mathrm{C}$ cell, including three acoustic modes and 21 optical modes. It is obvious from the figure that there is no virtual frequency in this doping system. It can therefore be concluded that the C-Ge codoped GaN system has dynamic stability.

\section{CONCLUSIONS}

The electronic properties of C monodoped, C-Ge, and C$\mathrm{Sn}$ codoped $\mathrm{GaN}$ nanosheets have been investigated at the HSE06 level of theory. For $\mathrm{C}$ monodoping, the effective band gap values of the $\mathrm{GaN}_{1-x} \mathrm{C}_{x}(\mathrm{x}=0.125)$ nanosheet can be decreased. However, the $\mathrm{C}$ monodoping will introduce the unoccupied impurity states, which leads to the decrease of carrier concentration. The band gap values for the C-Ge and C-Sn codoping GaN nanosheets are 1.83 and $1.85 \mathrm{eV}$, respectively. Narrow band gap semiconductors can absorb and utilize visible light, and semiconductors can thus be used in solar energy conversion and photocatalysis. The $\mathrm{C}-\mathrm{Ge}$ and $\mathrm{C}-\mathrm{Sn}$ codoping GaN nanosheets are direct band gap semiconductors, which make them more advantageous over other materials. Anionic (C) and cationic ( $\mathrm{Ge}$ or $\mathrm{Sn}$ ) compensated cooping, greatly reducing the minimum formation energy of doping. However, the minimum defect formation energy of the C-Ge codoping $\mathrm{GaN}$ nanosheet is the smallest. The $\mathrm{C}-\mathrm{Ge}$ and $\mathrm{C}-\mathrm{Sn}$ codoped systems may be a potential candidate for photocatalytic water splitting to generate hydrogen because of their appropriate band gap and band edge positions. More importantly, the result of the optical absorption spectral analysis shows that the C-Ge and C$\mathrm{Sn}$ codoped $\mathrm{GaN}$ nanosheets absorb a longer wavelength of the visible light spectrum as compared to the pure $\mathrm{GaN}$ nanosheet. Those results imply that the C-Ge codoping GaN nanosheet could be promising candidate as a visible light photocatalyst for water splitting.

\section{DATA AVAILABILITY STATEMENT}

All datasets generated for this study are included in the article/supplementary material.

\section{AUTHOR CONTRIBUTIONS}

CK: acquisition, analysis, and interpretation of data for the work. CT: drafting the work or revising it critically for important intellectual content. All authors contributed to the article and approved the submitted version. 


\section{REFERENCES}

Ahmad Khandy, S., and Chai, J.-D. (2020). Robust stability, half-metallic ferrimagnetism and thermoelectric properties of new quaternary Heusler material: a first principles approach. J. Magn. Magn. Mater. 502:166562. doi: 10.1016/j.jmmm.2020.166562

Ahmad Khandy, S., Islam, I., Gupta, D. C., Khenata, R., and Laref, A. (2019). Lattice dynamics, mechanical stability and electronic structure of Fe-based Heusler semiconductors. Sci. Rep. 9:1475. doi: 10.1038/s41598-018-37740-y

Armstrong, A., Arehart, A. R., Green, D., Mishra, U. K., and Ringel, S. A. (2005). Impact of deep levels on the electrical conductivity and luminescence of gallium nitride codoped with carbon and silicon. J. Appl. Phys. 98, 1145-1127. doi: $10.1063 / 1.2005379$

Bastos, C. M. O., Sabino, F. P., Sipahi, G. M., and Da Silva, J. L. F. (2018). A comprehensive study of $\mathrm{g}$-factors, elastic, structural and electronic properties of III-V semiconductors using hybrid-density functional theory. J. Appl. Phys. 123:065702. doi: 10.1063/1.5018325

Blöchl, P. E. (1994). Projector augmented-wave method. Phys. Rev. B Condens. Matter 50, 17953-17979. doi: 10.1103/PhysRevB.50.17953

Blöchl, P. E., Jepsen, O., and Andersen, O. K. (1994). Improved tetrahedron method for Brillouin-zone integrations. Phys. Rev. B 49, 16223-16233. doi: 10.1103/PhysRevB.49.16223

Castleton, C. W. M., Höglund, A., and Mirbt, S. (2006). Managing the supercell approximation for charged defects in semiconductors: finite-size scaling, charge correction factors, the band-gap problem, and the ab initio dielectric constant. Phys. Rev. B 73:035215. doi: 10.1103/PhysRevB.73.035215

Castro Neto, A. H., Guinea, F., Peres, N. M. R., Novoselov, K. S., and Geim, A. K. (2009). The electronic properties of graphene. Rev. Mod. Phys. 81, 109-162. doi: 10.1103/RevModPhys.81.109

Chakrapani, V., Angus, J. C., Anderson, A. B., Wolter, S. D., Stoner, B. R., and Sumanasekera, G. U. (2007). Charge transfer equilibria between diamond and an aqueous oxygen electrochemical redox couple. Science 318, 1424-1430. doi: $10.1126 /$ science. 1148841

Ernzerhof, M., and Scuseria, G. E. (1999). Assessment of the Perdew-BurkeErnzerhof exchange-correlation functional. J. Chem. Phys. 110, 5029-5036. doi: 10.1063/1.478401

Feng, L., Zhao, D., Jiang, D., Liu, Z., Zhu, J., Ping, C., et al. (2017). Suppression of optical field leakage to $\mathrm{GaN}$ substrate in $\mathrm{GaN}$-based green laser diode. Superlatt. Microstruct. 102, 484-489. doi: 10.1016/j.spmi.2017.01.012

Freeman, C. L., Claeyssens, F., Allan, N. L., Harding, J. H. (2006). Graphitic nanofilms as precursors to wurtzite films: theory. Phys. Rev. Lett. 96:066102. doi: 10.1103/PhysRevLett.96.066102

Gai, Y., Li, J., Li, S.-S., Xia, J.-B., and Wei, S.-H. (2009). Design of narrow-gap $\mathrm{TiO}_{2}$ : a passivated codoping approach for enhanced photoelectrochemical activity. Phys. Rev. Lett. 102:036402. doi: 10.1103/PhysRevLett.102.036402

Golberg, D., Bando, Y., Huang, Y., Terao, T., Mitome, M., Tang, C., et al. (2010). Boron nitride nanotubes and nanosheets. ACS Nano 4, 2979-2993. doi: $10.1021 / \mathrm{nn} 1006495$

Ha, G. Y., Park, T. Y., Kim, J. Y., Kim, D. J., Min, K. I., and Park, S. J. (2007). Improvement of reliability of GaN-based light-emitting diodes by selective wet etching with p-GaN. IEEE Photon. Technol. Lett. 19, 813-815. doi: 10.1109/LPT.2007.897291

Heyd, J., and Scuseria, G. E. (2004). Efficient hybrid density functional calculations in solids: assessment of the Heyd-Scuseria-Ernzerhof screened Coulomb hybrid functional. J. Chem. Phys. 121, 1187-1192. doi: 10.1063/1.1760074

Heyd, J., Scuseria, G. E., and Ernzerhof, M. (2003). Hybrid functionals based on a screened Coulomb potential. J. Chem. Phys. 118, 8207-8215. doi: $10.1063 / 1.1564060$

Kida, T., Minami, Y., Guan, G., Nagano, M., Akiyama, M., Yoshida, A., et al. (2006). Photocatalytic activity of gallium nitride for producing hydrogen from water under light irradiation. J. Mater. Sci. 41, 3527-3534. doi: $10.1007 / \mathrm{s} 10853-005-5655-8$

Kresse, G., and Furthmüller, J. (1996). Efficient iterative schemes for ab initio totalenergy calculations using a plane-wave basis set. Phys. Rev. B 54, 11169-11186. doi: 10.1103/PhysRevB.54.11169

Kresse, G., and Joubert, D. (1999). From ultrasoft pseudopotentials to the projector augmented-wave method. Phys. Rev. B 59, 1758-1775. doi: 10.1103/PhysRevB.59.1758

Maeda, K., Takata, T., Hara, M., Saito, N., Inoue, Y., Kobayashi, H., et al. (2005). GaN:ZnO solid solution as a photocatalyst for visible-light-driven overall water splitting. J. Am. Chem. Soc. 127, 8286-8287. doi: 10.1021/ja05 18777

Maruska, H. P., and Tietjen, J. J. (1969). The preparation and properties of vapor-deposited single-crystal-line GaN. Appl. Phys. Lett. 15, 327-329. doi: $10.1063 / 1.1652845$

Meng, G., Ying, D., and BaiBiao, H. (2012). Tailoring the band gap of GaN codoped by $\mathrm{VO}$ for enhanced solar energy conversion from first-principles calculations. Comput. Mater. Sci. 54, 101-104. doi: 10.1016/j.commatsci.2011. 10.019

Monkhorst, H. J., and Pack, J. D. (1976). Special points for Brillouin-zone integrations. Phys. Rev. B 13, 5188-5192. doi: 10.1103/PhysRevB.13.5188

Onen, A., Kecik, D., Durgun, E., Ciraci, S. (2016). GaN: from three- to twodimensional single-layer crystal and its multilayer van der Waals solids. Phys. Rev. B 93:085431. doi: 10.1103/PhysRevB.93.085431

Pan, H., Gu, B., Eres, G., Zhang, Z. (2010). Ab initio study on noncompensated $\mathrm{CrO}$ codoping of $\mathrm{GaN}$ for enhanced solar energy conversion. J. Chem. Phys. 132:104501. doi: 10.1063/1.3337919

Sahin, H., Cahangirov, S., Topsakal, M., Bekaroglu, E., Aktrk, E., Senger, R. T., et al. (2009). Monolayer honeycomb structures of group IV elements and III-V binary compounds. Phys. Rev. B 80:155453. doi: 10.1103/PhysRevB.80.155453

Sun, M., Chou, J. P., Yu, J., and Tang, W. (2017). Electronic properties of blue phosphorene/graphene and blue phosphorene/graphene-like gallium nitride heterostructures. Phys. Chem. Chem. Phys. 19:17324. doi: 10.1039/C7CP01852E

Van de Walle, C. G., and Neugebauer, J. (2004). First-principles calculations for defects and impurities: applications to III-nitrides. J. Appl. Phys. 95, 3851-3879. doi: $10.1063 / 1.1682673$

White, J. A., and Bird, D. M. (1994). Implementation of gradient-corrected exchange-correlation potentials in Car-Parrinello total-energy calculations. Phys. Rev. B 50, 4954-4957. doi: 10.1103/PhysRevB.50.4954

Xia, C., Peng, Y., Wei, S., and Jia, Y. U. (2013). The feasibility of tunable ptype $\mathrm{Mg}$ doping in a GaN monolayer nanosheet. Acta Mater. 61, 7720-7725. doi: 10.1016/j.actamat.2013.09.010

Xuan, F., Chai, J.-D., and Su, H. (2019). Local density approximation for the short-range exchange free energy functional. ACS Omega 4, 7675-7683. doi: 10.1021/acsomega.9b00303

Zeyan, W., Baibiao, H., Lin, Y., Ying, D., Peng, W., Xiaoyan, Q., et al. (2008). Enhanced ferromagnetism and tunable saturation magnetization of $\mathrm{Mn} / \mathrm{C}$ codoped GaN nanostructures synthesized by carbothermal nitridation. J. Am. Chem. Soc. 130:16366. doi: 10.1021/ja807030y

Conflict of Interest: The authors declare that the research was conducted in the absence of any commercial or financial relationships that could be construed as a potential conflict of interest.

Copyright (c) $2020 \mathrm{Ke}$, Tian and Gan. This is an open-access article distributed under the terms of the Creative Commons Attribution License (CC BY). The use, distribution or reproduction in other forums is permitted, provided the original author(s) and the copyright owner(s) are credited and that the original publication in this journal is cited, in accordance with accepted academic practice. No use, distribution or reproduction is permitted which does not comply with these terms. 